Kragujevac Journal of Mathematics

Volume 38(1) (2014), Pages 105-123.

\title{
UNIQUENESS OF MEROMORPHIC FUNCTIONS SHARING THREE VALUES
}

\author{
ARINDAM SARKAR ${ }^{1}$ AND PAULOMI CHATTOPADHYAY ${ }^{2}$
}

\begin{abstract}
We prove four uniqueness theorems for meromorphic functions $f$ and $g$ sharing values $0,1, \infty$ which improve results of I. Lahiri, X. M. Li, H. X. Yi, C. Meng and others.
\end{abstract}

\section{Introduction, Definitions and Results}

Let $f$ and $g$ be two non constant meromorphic functions defined in the open complex plane $\mathbb{C}$. If for some $a \in \mathbb{C} \cup\{\infty\}, f$ and $g$ have the same set of $a$-points with the same multiplicities we say that $f$ and $g$ share the value $a$ CM (Counting Multiplicities) and if we do not consider the multiplicities, then $f$ and $g$ are said to share the value $a$ IM (Ignoring Multiplicities). We do not explain the standard notations and definitions of the value distribution theory as these are available in [2]. It will be convenient to let $E$ denote any set of positive real numbers of finite linear measure, not necessary the same at each occurrence. For a non-constant meromorphic function $h$, we denote by $T(r, h)$ the Nevanlinna characteristic of $h$ and by $S(r, h)$ any quantity satisfying $S(r, h)=o\{T(r, h)\}$, as $r \rightarrow \infty$ and $r \notin E$.

Definition 1.1. [1] Let $p$ be a positive integer and $a \in \mathbb{C} \cup\{\infty\}$. We denote by $\bar{N}(r, a ; f \mid=p)$ the counting function of those $a$-points of $f$ whose multiplicities are exactly equal to $p$, where an $a$-point is counted only once.

In 1976, Ozawa [17] proved the following theorem.

Theorem 1.1. [17] Let $f$ and $g$ be two non constant entire functions of finite order. If $f, g$ share $0,1 C M$ and $2 \delta(0, f)>1$ then either $f \equiv g$ or $f g \equiv 1$.

Key words and phrases. Meromorphic functions, Uniqueness, Weighted sharing.

2010 Mathematics Subject Classification. Primary: 30D35.

Received:November 5, 2012

Revised: July 29, 2013. 
Ueda [18] extended the above result to meromorphic functions and freed it from order restriction in the following theorem.

Theorem 1.2. [18] Let $f, g$ share $0,1, \infty C M$. If

$$
\limsup _{r \rightarrow \infty} \frac{N(r, 0 ; f)+N(r, \infty ; f)}{T(r, f)}<\frac{1}{2}
$$

then either $f \equiv g$ or $f g \equiv 1$.

Yi further improved Theorem 1.2 and proved the following.

Theorem 1.3. [19] Let $f, g$ share $0,1, \infty$ CM. If

$$
\bar{N}(r, 0 ; f \mid=1)+\bar{N}(r, \infty ; f \mid=1)<\{\lambda+o(1)\} T(r, f)
$$

for $r \in I$, where $0<\lambda<\frac{1}{2}$ and $I$ is a set of infinite linear measure, then either $f \equiv g$ or $f g \equiv 1$.

We recall the following example from Lahiri [9]: If $f=\left(e^{z}-1\right)^{2}$ and $g=e^{z}-1$, then we see that in Theorem 1.3 the sharing of 0 cannot be relaxed from CM to IM.

In 2001 Lahiri [5] considered the following problem: Is it possible to relax the nature of sharing of the value 0 in Theorem 1.3 and if possible how far? To this end Lahiri employed the notion of weighted sharing introduced by him in 2001 expressed in the following definition.

Definition 1.2. $[4,5]$ Let $k$ be a nonnegative integer or infinity. For $a \in \mathbb{C} \cup\{\infty\}$ we denote by $E_{k}(a ; f)$ the set of all $a$-points of $f$ where an $a$-point of multiplicity $m$ is counted $m$ times if $m \leq k$ and $k+1$ times if $m>k$. If $E_{k}(a ; f)=E_{k}(a ; g)$, we say that $f$ and $g$ share the value $a$ with weight $k$.

The definition implies that if $f, g$ share a value $a$ with weight $k$, then $z_{0}$ is a zero of $f-a$ with multiplicity $m(\leq k)$ if and only if it is a zero of $g-a$ with multiplicity $m(\leq k)$ and $z_{0}$ is a zero of $f-a$ of multiplicity $m(>k)$ if and only if it is a zero of $g-a$ with multiplicity $n(>k)$ where $m$ is not necessarily equal to $n$.

We write $f, g$ share $(a, k)$ to mean $f, g$ share the value $a$ with weight $k$. Clearly if $f, g$ share $(a, k)$ then $f, g$ share $(a, p)$ for all integers $p, 0 \leq p<k$. Also we note that $f, g$ share a value $a$ IM or CM if and only if $f, g$ share $(a, 0)$ or $(a, \infty)$ respectively.

Below we state Lahiri's result which improves Theorem 1.3.

Theorem 1.4. [5] Let $f, g$ share $(0,1),(1, \infty),(\infty, \infty)$. If

$$
\bar{N}(r, 0 ; f \mid=1)+\bar{N}(r, \infty ; f \mid=1)<\{\lambda+o(1)\} T(r, f)
$$

for $r \in I$, where $0<\lambda<\frac{1}{2}$ and $I$ is a set of infinite linear measure then either $f \equiv g$ or $f g \equiv 1$.

We need the following definitions to proceed further. 
Definition 1.3. [8] Let $p$ be a positive integer and $a \in \mathbb{C} \cup\{\infty\}$. We denote by $N(r, a ; f \mid \geq p),(\bar{N}(r, a ; f \mid \geq p))$ the counting function(reduced counting function) of those $a$-points of $f$ whose multiplicities are greater than or equal to $p$, where an $a$-point is counted according to its multiplicity (only once). Similarly we denote by $N(r, a ; f \mid \leq p),(\bar{N}(r, a ; f \mid \leq p))$ the counting function(reduced counting function) of those $a$-points of $f$ whose multiplicities are less than or equal to $p$, where an $a$-point is counted according to its multiplicity (only once).

Definition 1.4. For $a \in \mathbb{C} \cup\{\infty\}$, we define

$$
\begin{aligned}
\Theta(a ; f) & =1-\limsup _{r \rightarrow \infty} \frac{\bar{N}(r, a ; f)}{T(r, f)} \\
\delta_{1)}(a ; f) & =1-\limsup _{r \rightarrow \infty} \frac{N(r, a ; f \mid=1)}{T(r, f)} ; \\
\delta_{2)}(a ; f) & =1-\limsup _{r \rightarrow \infty} \frac{N(r, a ; f \mid \leq 2)}{T(r, f)} ; \\
\delta_{2}(a, f) & =1-\limsup _{r \rightarrow \infty} \frac{N_{2}(r, a ; f)}{T(r, f)}
\end{aligned}
$$

where by $N_{2}(r, a ; f)$ we denote the $\operatorname{sum} \bar{N}(r, a ; f)+\bar{N}(r, a ; f \mid \geq 2)$.

Following are the chronological improvements by Lahiri of Theorem 1.4 in 2002 and 2003 respectively.

Theorem 1.5. [8] If $f$ and $g$ are two non constant meromorphic functions sharing $(0,1),(1, \infty),(\infty, \infty)$ and

$$
2 \delta_{1)}(0 ; f)+2 \delta_{1)}(\infty ; f)+\min \left\{\sum_{a \neq 0,1, \infty} \delta_{2)}(a ; f), \sum_{a \neq 0,1, \infty} \delta_{2)}(a, g)\right\}>3
$$

then either $f \equiv g$ or $f g \equiv 1$. If $f$ has at least one zero or pole the case $f g \equiv 1$ dose not arise.

Theorem 1.6. [9] Theorem 1.5 holds if $f, g$ share $(0,1),(1, m),(\infty, k)$, where

$$
(m-1)(m k-1)>(1+m)^{2} .
$$

Regarding Theorem 1.6 it is natural to raise the following questions.

Question 1.1. What can be said about the relationship between $f$ and $g$ if we relax in any way the condition (1.1)?

Question 1.2. What can be said about the relationship, if we relax the nature of sharing of the values $0,1, \infty$ in any way?

In this direction following results were established with gradual improvements by Q. C. Zhang [25] in 2006, by C. Meng [15] in 2008 and most recently by Li-Yi [14] in 2010 . 
Theorem 1.7. [25] Let $f$ and $g$ be two nonconstant meromorphic functions sharing $\left(a_{1}, k_{1}\right),\left(a_{2}, k_{2}\right)$ and $\left(a_{3}, k_{3}\right)$ where $\left\{a_{1}, a_{2}, a_{3}\right\}=\{0,1, \infty\}$ and $k_{1}, k_{2}, k_{3}$ are three positive integers satisfying the condition

$$
k_{1} k_{2} k_{3}>k_{1}+k_{2}+k_{3}+2 \text {. }
$$

If

$$
2 \delta_{1)}(0 ; f)+2 \delta_{1)}(\infty ; f)+\sum_{a \neq 0,1, \infty} \delta_{2)}(a ; f)+\max \left\{\delta_{1)}(1, f), \delta_{1)}(1, g)\right\}>3
$$

and

$$
2 \delta_{1)}(0 ; g)+2 \delta_{1)}(\infty ; g)+\sum_{a \neq 0,1, \infty} \delta_{2)}(a ; g)+\max \left\{\delta_{1)}(1, f), \delta_{1)}(1, g)\right\}>3,
$$

then either $f \equiv g$ or $f g \equiv 1$.

Theorem 1.8. [25] Let $f$ and $g$ be two transcendental meromorphic functions such that $f$ and $g$ share $\left(0, k_{1}\right),\left(1, k_{2}\right),\left(\infty, k_{3}\right)$ where $k_{1}, k_{2}, k_{3}$ are three positive integers satisfying (1.2). If both

$2 \delta_{1)}(0 ; f)+2 \delta_{1)}(\infty ; f)+\sum_{a \neq 0,1, \infty} \delta_{2)}(a ; f)+\sum_{a \neq 0,1, \infty} \delta_{2)}(a ; g)+\max \left\{\delta_{1)}(1, f), \delta_{1)}(1, g)\right\}>3$,

and

$2 \delta_{1)}(0 ; g)+2 \delta_{1)}(\infty ; g)+\sum_{a \neq 0,1, \infty} \delta_{2)}(a ; f)+\sum_{a \neq 0,1, \infty} \delta_{2)}(a ; g)+\max \left\{\delta_{1)}(1, f), \delta_{1)}(1, g)\right\}>3$, hold, then one of the following four equalities hold:

(i) $f \equiv g$;

(ii) $f g \equiv 1$;

(iii) $f-1 \equiv A(g-1)$;

(iv) $\frac{1}{f}-1 \equiv A\left(\frac{1}{g}-1\right)$;

where $A(\neq 0,1)$ is a finite complex number.

Theorem 1.9. [15] Let $f$ and $g$ be two non constant meromorphic functions sharing $(0, m),(\infty, 0)$ and $(1,1)$ where $m \geq 2$. If

$$
2 \delta_{2}(0 ; f)+\frac{4 m}{m-1} \delta_{2}(\infty ; f)+\min \left\{\sum_{a \neq 0,1, \infty} \delta_{2}(a ; f), \sum_{a \neq 0,1, \infty} \delta_{2}(a, g)\right\}>\frac{5 m-1}{m-1},
$$

then either $f \equiv g$ or $f g \equiv 1$.

Corollary 1.1. Let $f$ and $g$ be two non constant meromorphic functions sharing $(0,2),(\infty, 0)$ and $(1,1)$. If

$$
2 \delta_{2}(0 ; f)+8 \delta_{2}(\infty ; f)+\min \left\{\sum_{a \neq 0,1, \infty} \delta_{2}(a ; f), \sum_{a \neq 0,1, \infty} \delta_{2}(a, g)\right\}>9,
$$

then either $f \equiv g$ or $f g \equiv 1$. 
Corollary 1.2. [15] Let $f$ and $g$ be two non constant meromorphic functions sharing $(0,3),(\infty, 0)$ and $(1,1)$. If

$$
2 \delta_{2}(0 ; f)+6 \delta_{2}(\infty ; f)+\min \left\{\sum_{a \neq 0,1, \infty} \delta_{2}(a ; f), \sum_{a \neq 0,1, \infty} \delta_{2}(a, g)\right\}>7,
$$

then either $f \equiv g$ or $f g \equiv 1$.

Theorem 1.10. [14] Let $f$ and $g$ be two nonconstant entire functions such that $f$ and $g$ share $\left(a_{1}, 1\right),\left(a_{2}, 2\right)$ where $\left\{a_{1}, a_{2}\right\}=\{0,1\}$. If

$$
2 \delta_{1)}(0 ; f)+\max \left\{\sum_{a \neq 0,1, \infty} \delta_{2)}(a ; f), \sum_{a \neq 0,1, \infty} \delta_{2)}(a, g)\right\}+\max \left\{\delta_{1)}(1, f), \delta_{1)}(1, g)\right\}>1,
$$

then either $f \equiv g$ or $f g \equiv 1$.

Theorem 1.11. [14] Let $f$ and $g$ be two transcendental meromorphic functions such that $f$ and $g$ share $\left(0, k_{1}\right),\left(1, k_{2}\right),\left(\infty, k_{3}\right)$ where $k_{1}, k_{2}, k_{3}$ are three positive integers satisfying (1.2). If

$$
2 \delta_{1)}(0 ; f)+2 \delta_{1)}(\infty ; f)+\max \left\{\sum_{a \neq 0,1, \infty} \delta_{2)}(a ; f), \sum_{a \neq 0,1, \infty} \delta_{2)}(a, g)\right\}>3
$$

then either $f \equiv g$ or $f g \equiv 1$.

Theorem 1.12. [14] Let $f$ and $g$ be two transcendental meromorphic functions such that $f$ and $g$ share $\left(0, k_{1}\right),\left(1, k_{2}\right),\left(\infty, k_{3}\right)$ where $k_{1}, k_{2}, k_{3}$ are three positive integers satisfying $k_{1} k_{2} k_{3}>k_{1}+k_{2}+k_{3}+2$. If

$$
\begin{aligned}
& 2 \delta_{1)}(0 ; f)+2 \delta_{1)}(\infty ; f)+2 \delta_{1)}(0 ; g)+2 \delta_{1)}(\infty ; g)+\sum_{a \neq 0,1, \infty} \delta_{2)}(a ; f) \\
& +\sum_{a \neq 0,1, \infty} \delta_{2)}(a ; g)+\delta_{1)}(1, f)+\delta_{1)}(1, g)>6
\end{aligned}
$$

hold, then either $f \equiv g$ or $f g \equiv 1$.

Theorem 1.13. [15] Let $f$ and $g$ be two transcendental meromorphic functions such that $f$ and $g$ share $\left(0, k_{1}\right),\left(1, k_{2}\right),\left(\infty, k_{3}\right)$, where $k_{1}, k_{2}, k_{3}$ are three positive integers satisfying (1.2). If

$$
\begin{aligned}
& 2 \delta_{1)}(0 ; f)+2 \delta_{1)}(\infty ; f)+\max \left\{\sum_{a \neq 0,1, \infty} \delta_{2)}(a ; f), \sum_{a \neq 0,1, \infty} \delta_{2)}(a ; g)\right\}+\delta_{1)}(1, f) \\
& +\delta_{1)}(1, g)>3
\end{aligned}
$$


and

$$
\begin{aligned}
& 2 \delta_{1)}(0 ; g)+2 \delta_{1)}(\infty ; g)+\max \left\{\sum_{a \neq 0,1, \infty} \delta_{2)}(a ; f), \sum_{a \neq 0,1, \infty} \delta_{2)}(a ; g)\right\}+\delta_{1)}(1, f) \\
& +\delta_{1)}(1, g)>3
\end{aligned}
$$

hold, then one of the following four equalities holds:

(i) $f \equiv g$;

(ii) $f g \equiv 1$;

(iii) $(f-1)(g-1) \equiv 1$;

(iv) $f+g \equiv 1$.

Remark 1.1. Theorem 1.6 holds for $m k \geq 12$, where $m \geq 2$ and $k \geq 2$. A set of possible values of $m$ and $k$ may be as follows: (i) $m=2, k=6$, (ii) $m=3, k=$ 4, (iii) $m=4, k=3$, (iv) $m=6, k=2$. Similarly in Theorem 1.7 or Theorem 1.11 if $k_{1}=1$, then $k_{2}, k_{3}$ must be at least 2 and 6 or 3 and 4 or 4 and 3 or 6 and 2 respectively. Therefore it is natural to investigate situation under which the conclusions of Theorems 1.6 and 1.7 or 1.11 are true while $m=1, k=1$ or $k_{1}=k_{2}=$ $k_{3}=1$ respectively.

Thus above theorems lead us to the following questions.

Question 1.3. What can be said about the conclusion of Theorem 1.9, if $m=1$ and if we relax the assumption 1.3 in any way?

Question 1.4. What can be said about the conclusions of Theorems 1.6 and 1.7 , if $f$ and $g$ share $(0,1),(1,1),(\infty, 1)$ ?

Question 1.5. What can be said about the conclusions of Theorem 1.11, if $f$ and $g$ are non-constant meromorphic functions and if we relax the assumption 1.5 in any way?

These questions are the motivations of this paper, which we answer in affirmative. Next we state the main results, where Theorem 1.14 answers Question 1.4,Theorem 1.15 answers Question 1.5 and Question 1.3 is answered by Theorem 1.16 that is a two-fold improvement of Theorem 1.9 as it is proved under the assumption $m=1$ and a weaker assumption than condition (1.3). Finally we give Theorem 1.17 which supplements Theorem 1.13.

Theorem 1.14. Let $f$ and $g$ be two nonconstant meromorphic functions sharing $(0,1),(1,1),(\infty, 1)$. If

$$
A_{1}=2 \delta_{2}(0 ; f)+2 \delta_{2}(\infty ; f)+\sum_{a \neq 0,1, \infty} \delta_{2}(a ; f)+\liminf _{r \rightarrow \infty} \frac{m(r, 1 ; g)}{T(r, f)}>3,
$$

then either $f \equiv g$ or $f g \equiv 1$. 
Theorem 1.15. Let $f$ and $g$ be two nonconstant meromorphic functions sharing $\left(0, k_{1}\right),\left(1, k_{2}\right),\left(\infty, k_{3}\right)$ where $k_{1} k_{2} k_{3}>k_{1}+k_{2}+k_{3}+2$. If

$$
\begin{aligned}
A_{2}= & 2 \delta_{1)}(0 ; f)+2 \delta_{1)}(\infty ; f)+\max \left\{\sum_{a \neq 0,1, \infty} \delta_{2)}(a ; f), \sum_{a \neq 0,1, \infty} \delta_{2}(a ; g)\right\} \\
& +\max \left\{\delta_{1)}(1 ; f), \delta_{1)}(1 ; g)\right\}>3,
\end{aligned}
$$

then either $f \equiv g$ or $f g \equiv 1$.

Theorem 1.16. Let $f$ and $g$ be two nonconstant meromorphic functions sharing $(0,1),(1,1),(\infty, 0)$. If

$$
A_{3}=2 \delta_{2}(0 ; f)+4 \Theta(\infty ; f)+\sum_{a \neq 0,1, \infty} \delta_{2}(a ; f)+\liminf _{r \rightarrow \infty} \frac{m(r, 1 ; g)}{T(r, f)}>5
$$

then either $f \equiv g$ or $f g \equiv 1$.

Theorem 1.17. Let $f$ and $g$ be two nonconstant meromorphic functions such that $f$ and $g$ share $\left(0, k_{1}\right),\left(1, k_{2}\right),\left(\infty, k_{3}\right)$ where $k_{1}, k_{2}, k_{3}$ are three positive integers satisfying (1.2). If

$$
\begin{aligned}
& 2 \delta_{1)}(0 ; f)+2 \delta_{1)}(\infty ; f)+\sum_{a \neq 0,1, \infty} \delta_{2)}(a ; f)+\sum_{a \neq 0,1, \infty} \delta_{2)}(a ; g)+\delta_{1)}(1, f) \\
& +\delta_{1)}(1, g)>3
\end{aligned}
$$

and

$$
\begin{aligned}
& 2 \delta_{1)}(0 ; g)+2 \delta_{1)}(\infty ; g)+\sum_{a \neq 0,1, \infty} \delta_{2)}(a ; f)+\sum_{a \neq 0,1, \infty} \delta_{2)}(a ; g)+\delta_{1)}(1, f) \\
& +\delta_{1)}(1, g)>3
\end{aligned}
$$

hold, then $f$ is a bilinear transformation of $g$ and one of the following equalities holds:

(i) $f \equiv g$;

(ii) $f g \equiv 1$;

(iii) $(f-1)(g-1) \equiv 1$;

(iv) $f+g \equiv 1$;

(v) $f \equiv c g$;

(vi) $f-1 \equiv c(g-1)$;

(vii) $[(c-1) f+1][(c-1) g-c]+c \equiv 1$, where $c(\neq 0,1, \infty)$

is a constant.

From Theorem 1.14 we obtain the following corollary.

Corollary 1.3. Let $f$ and $g$ be two nonconstant entire functions sharing $(0,1),(1,1)$. If

$$
2 \delta_{2}(0 ; f)+\sum_{a \neq 0,1, \infty} \delta_{2}(a ; f)+\liminf _{r \rightarrow \infty} \frac{m(r, 1 ; g)}{T(r, f)}>1
$$


then either $f \equiv g$ or $f g \equiv 1$.

We illustrate some examples to show that the condition in Theorem 1.14 is sharp. Example 1.1. [7] Let $f=e^{z}-1$ and $g=2-\frac{2}{e^{z}}$. Then $f$ and $g$ share $(0, \infty),(1, \infty)$, $(\infty, \infty)$ and therefore $(0,1),(1,1),(\infty, 1)$. An easy calculation shows that $A_{1}=3$, but neither $f \equiv g$ or $f g \equiv 1$.

Example 1.2. Let $f=\frac{4 e^{z}\left(e^{z}-1\right)^{2}}{\left(e^{z}+1\right)^{4}}, g=-\frac{\left(e^{z}-1\right)^{4}}{4 e^{z}\left(e^{z}+1\right)^{2}}$. Clearly $f, g$ share $(0,1),(\infty, 1)$ and $(1, \infty)$ and $T(r, f)=4 T\left(r, e^{z}\right)+O(1), \bar{N}(r, \infty ; f \mid \geq 2) \sim T\left(r, e^{z}\right), \bar{N}(r, \infty ; f) \sim$ $T\left(r, e^{z}\right), \bar{N}(r, 0 ; f \mid \geq 2) \sim T\left(r, e^{z}\right), \bar{N}(r, 0 ; f) \sim T\left(r, e^{z}\right), N(r, 1 ; g) \sim 4 T\left(r, e^{z}\right)$. Therefore $\delta_{2}(0, f)=\delta_{2}(\infty, f)=\frac{1}{2}$. Similarly $\delta_{2}(1, f)=\frac{1}{2}$. Thus the maximum value of $\sum_{a \neq 0,1, \infty} \delta_{2}(a ; f)$ is $2-\frac{3}{2}=\frac{1}{2}$. Therefore the maximum value of $A_{1}=\frac{5}{2}$ and we observe that neither $f \equiv g$ nor $f g \equiv 1$.

Remark 1.2. From Theorem 1.15 we can get Theorem 1.10 directly.

Remark 1.3. We first notice that the inequality (1.4) in Corollary 1.1 can be rewritten as $2 \delta_{2}(0 ; f)+4 \delta_{2}(\infty ; f)+\min \left\{\sum_{a \neq 0,1, \infty} \delta_{2}(a ; f), \sum_{a \neq 0,1, \infty} \delta_{2}(a, g)\right\}>5+4(1-$ $\left.\delta_{2}(\infty ; f)\right)$. This implies that our Theorem 1.16 is a two-fold improvement of Corollary 1.1. One is due to reduction of weight and another is due to weaker condition than (1.4). Similar conclusion can be drawn regarding Corollary 1.2 and Theorem 1.16.

Definition 1.5. Let $f$ and $g$ share the value 1 IM. Let $z_{0}$ be a 1-point of $f$ and $g$ with multiplicities $p$ and $q$ respectively. Let $s$ be a positive integer. We denote by $\bar{N}_{f>s}(r, 1 ; g)$ the reduced counting function of those 1-points of $f$ and $g$ such that $p>q=s$. Similarly $\bar{N}_{f<s}(r, 1 ; g)$ will denote the reduced counting function of those 1-points of $f$ and $g$ where $p<q=s$.

Definition 1.6. Let $f$ and $g$ be two nonconstant meromorphic functions such that $f$ and $g$ share $(a, k)$ where $a \in \mathbb{C} \cup\{\infty\}$. Let $z_{0}$ be an $a$-point of $f$ with multiplicity $p$, an $a$-point of $g$ of multiplicity $q$. We denote by $\bar{N}_{L}(r, a ; f)\left(\bar{N}_{L}(r, a ; g)\right)$ the counting function of those $a$-points of $f$ and $g$ where $p>q(q>p)$, by $\bar{N}_{E}^{(k+1}(r, a ; f)$ the counting functions of those $a$-points of $f$ and $g$ where $p=q \geq k+1$ each point in these counting functions is counted only once. In the same way we can define $\bar{N}_{E}^{(k+1}(r, a ; g)$. Clearly $\bar{N}_{E}^{(k+1}(r, a ; f)=\bar{N}_{E}^{(k+1}(r, a ; g)$.

Definition 1.7. [4, 5] Let $f, g$ share the value $a$ IM. We denote by $\bar{N}_{*}(r, a ; f, g)$ the reduced counting function of those $a$-points of $f$ whose multiplicities differ from the corresponding $a$-points of $g$. Clearly $\bar{N}_{*}(r, a ; f, g)=\bar{N}_{*}(r, a ; g, f)$ and $\bar{N}_{*}(r, a ; f, g)=$ $\bar{N}_{L}(r, a ; f)+\bar{N}_{L}(r, a ; g)$

Definition 1.8. Let $f, g$ share $(1,0)$ and $z_{0}$ be a common 1-point of $f$ and $g$ with multiplicities $p$ and $q$ respectively. By $\bar{N}_{f<g}(r, 1 ; g \mid \geq 4)$, we denote the reduced counting function of those 1-points of $f$ and $g$ such that $q \geq 4$ and $p<q$. Similarly $\bar{N}_{f>g}(r, 1 ; g \mid \geq 3)$ denote the reduced counting function of those common 1-points of $f$ and $g$ with $q \geq 3$ and $p>q$. 


\section{LEMMAS}

In this section we present some lemmas which will be needed in the sequel. In the lemmas several times we use the function $H$ defined by $H=\frac{f^{\prime \prime}}{f^{\prime}}-\frac{2 f^{\prime}}{f-1}-\frac{g^{\prime \prime}}{g^{\prime}}+\frac{2 g^{\prime}}{g-1}$.

Lemma 2.1. [3] If $f, g$ share $(0,0),(1,0),(\infty, 0)$ then

(i) $T(r, f) \leq 3 T(r, g)+S(r, f)$,

(ii) $T(r, g) \leq 3 T(r, f)+S(r, g)$.

Lemma 2.1 shows that $S(r, f)=S(r, g)$ and we denote it by $S(r)$.

Lemma 2.2. If $f$ and $g$ share $(1,1)$ then

$$
\begin{aligned}
N(r, 1 ; g)-\bar{N}(r, 1 ; g) \geq & 2 \bar{N}_{L}(r, 1 ; g)+\bar{N}_{L}(r, 1 ; f)+\bar{N}_{E}^{(2}(r, 1 ; f)+\bar{N}_{E}^{(3}(r, 1 ; f) \\
& +\bar{N}_{f<g}(r, 1 ; g \mid \geq 4)+\bar{N}_{f>g}(r, 1 ; g \mid \geq 3) .
\end{aligned}
$$

Proof. Let $z_{0}$ be a 1-point of $f$ and $g$ of multiplicities $p$ and $q$ respectively. We denote by $N_{1}(r), N_{2}(r)$ and $N_{3}(r)$ the counting functions of those 1-points of $f$ and $g$ with $2 \leq q<p, 2 \leq q=p$ and $2 \leq p<q$ respectively where each point in these counting functions is counted $q-2$ times. Since $f, g$ share $(1,1)$ we have $N(r, 1 ; g)-\bar{N}(r, 1 ; g)=\bar{N}_{E}^{(2}(r, 1 ; f)+N_{2}(r)+\bar{N}_{L}(r, 1 ; g)+N_{3}(r)+\bar{N}_{L}(r, 1 ; f)+N_{1}(r)$. Now $N_{3}(r) \geq \bar{N}_{L}(r, 1 ; g)+\bar{N}_{f<g}(r, 1 ; g \mid \geq 4), N_{2}(r) \geq \bar{N}_{E}^{(3}(r, 1 ; f)$ and $N_{1}(r) \geq$ $\bar{N}_{f>g}(r, 1 ; g \mid \geq 3)$ and the lemma follows from above.

Lemma 2.3. [7] If $f$ and $g$ share $(1,1)$ and $H \not \equiv 0$ then $N(r, 1 ; f \mid=1)=N(r, 1 ; g \mid=$ 1) $\leq N(r, H)+S(r, f)+S(r, g)$.

Lemma 2.4. [10] If $f$ and $g$ share $(0,0),(1,0),(\infty, 0)$ and $H \not \equiv 0$ then

$$
N(r, H) \leq \bar{N}_{*}(r, 0 ; f, g)+\bar{N}_{*}(r, 1 ; f, g)+\bar{N}_{*}(r, \infty ; f, g)+\bar{N}_{0}\left(r, 0 ; f^{\prime}\right)+\bar{N}_{0}\left(r, 0 ; g^{\prime}\right),
$$

where $\bar{N}_{0}\left(r, 0 ; f^{\prime}\right)$ is the reduced counting function of those zeros of $f^{\prime}$ which are not the zeros of $f(f-1)$ and $\bar{N}_{0}\left(r, 0 ; g^{\prime}\right)$ is similarly defined.

Lemma 2.5. If $f$ and $g$ share $(0,0),(1,1),(\infty, k)$ and $H \not \equiv 0$ then

$$
\begin{aligned}
\bar{N}(r, 1 ; f)+\bar{N}(r, 1 ; g) \leq & \bar{N}_{*}(r, 0 ; f, g)+\bar{N}_{*}(r, \infty ; f, g)+\bar{N}_{0}\left(r, 0 ; f^{\prime}\right)+\bar{N}_{0}\left(r, 0 ; g^{\prime}\right) \\
& +T(r ; g)-m(r, 1 ; g)-\bar{N}_{L}(r, 1 ; g)-\bar{N}_{E}^{(3}(r, 1 ; f) \\
& +\bar{N}_{g>2}(r, 1 ; f)+\bar{N}_{f>2}(r, 1 ; g)+S(r)
\end{aligned}
$$

where $\bar{N}_{0}\left(r, 0 ; f^{\prime}\right)$ and $\bar{N}_{0}\left(r, 0 ; g^{\prime}\right)$ are same as Lemma 2.4. 
Proof. By Lemmas 2.3, 2.4 and 2.2 we have

$$
\begin{aligned}
\bar{N}(r, 1 ; f)+\bar{N}(r, 1 ; g) \leq & N(r, H)+\bar{N}(r, 1 ; g)+\bar{N}(r, 1 ; f \mid \geq 2)+S(r) \\
\leq & \bar{N}_{*}(r, 0 ; f, g)+\bar{N}_{*}(r, 1 ; f, g)+\bar{N}_{*}(r, \infty ; f, g)+\bar{N}_{0}\left(r, 0 ; f^{\prime}\right) \\
& +\bar{N}_{0}\left(r, 0 ; g^{\prime}\right)+\bar{N}(r, 1 ; g)+\bar{N}(r, 1 ; f \mid \geq 2)+S(r) \\
\leq & \bar{N}_{*}(r, 0 ; f, g)+\bar{N}_{*}(r, 1 ; f, g)+\bar{N}_{*}(r, \infty ; f, g)+\bar{N}_{0}\left(r, 0 ; f^{\prime}\right) \\
& +\bar{N}_{0}\left(r, 0 ; g^{\prime}\right)+N(r, 1 ; g)-2 \bar{N}_{L}(r, 1 ; g)-\bar{N}_{L}(r, 1 ; f) \\
& -\bar{N}_{E}^{(2}(r, 1 ; f)-\bar{N}_{E}^{(3}(r, 1 ; f)-\bar{N}_{f<g}(r, 1 ; g \mid \geq 4) \\
& -\bar{N}_{f>g}(r, 1 ; g \mid \geq 3)+\bar{N}(r, 1 ; f \mid \geq 2)+S(r) .
\end{aligned}
$$

Now we observe that $f, g$ share $(1,1)$ and $\bar{N}(r, 1 ; f \mid \geq 2)=\bar{N}_{L}(r, 1 ; g)+\bar{N}_{L}(r, 1 ; f)+$ $\bar{N}_{E}^{(2}(r, 1 ; f)$, and $\bar{N}_{L}(r, 1 ; g)-\bar{N}_{f<g}(r, 1 ; g \mid \geq 4) \leq \bar{N}_{g>2}(r, 1 ; f)$ and $\bar{N}_{L}(r, 1 ; f)-$ $\bar{N}_{f>g}(r, 1 ; g \mid \geq 3) \leq \bar{N}_{f>2}(r, 1 ; g)$. Thus from above we obtain

$$
\begin{aligned}
\bar{N}(r, 1 ; f)+\bar{N}(r, 1 ; g) \leq & \bar{N}_{*}(r, 0 ; f, g)+\bar{N}_{*}(r, \infty ; f, g)-\bar{N}_{L}(r, 1 ; g)+T(r, g) \\
& -m(r, 1 ; g)+\bar{N}_{0}\left(r, 0 ; g^{\prime}\right)+\bar{N}_{0}\left(r, 0 ; f^{\prime}\right)-\bar{N}_{E}^{(3}(r, 1 ; f) \\
& +\bar{N}_{g>2}(r, 1 ; f)+\bar{N}_{f>2}(r, 1 ; g)+S(r) .
\end{aligned}
$$

This completes the proof.

Lemma 2.6. If $f$ and $g$ share $(0,1),(1,1),(\infty, k)$ and $H \not \equiv 0$, then

(i) $\bar{N}_{*}(r, 0 ; f, g) \leq \bar{N}(r, 0 ; f \mid \geq 2) \leq \bar{N}_{*}(r, 1 ; f, g)+\bar{N}(r, \infty ; f \mid \geq k+1)$,

(ii) $\bar{N}_{*}(r, 1 ; f, g) \leq \bar{N}(r, 0 ; f \mid \geq 2)+\bar{N}(r, \infty ; f \mid \geq k+1)$.

Proof. Let $\phi_{1}=\frac{f^{\prime}}{f-1}-\frac{g^{\prime}}{g-1}, \phi_{2}=\frac{f^{\prime}}{f}-\frac{g^{\prime}}{g}$. Since $H \not \equiv 0$, we have $f \not \equiv g$ and hence it follows that $\phi_{i} \not \equiv 0$ for $i=1,2$. Now

$$
\begin{aligned}
\bar{N}_{*}(r, 0 ; f, g) & \leq \bar{N}(r, 0 ; f \mid \geq 2) \leq N\left(r, 0 ; \phi_{1}\right) \leq T(r, f)+O(1)=N\left(r, \infty ; \phi_{1}\right)+S(r) \\
& \leq \bar{N}_{*}(r, 1 ; f, g)+\bar{N}(r, \infty ; f \mid \geq k+1)+S(r)
\end{aligned}
$$

which is (i). Again

$$
\begin{aligned}
\bar{N}_{*}(r, 1 ; f, g) & \leq N\left(r, 0 ; \phi_{2}\right) \leq T\left(r, \phi_{2}\right)+S(r)=N\left(r, \infty ; \phi_{2}\right)+S(r) \\
& \leq \bar{N}(r, 0 ; f \mid \geq 2)+\bar{N}(r, \infty ; f \mid \geq k+1)+S(r),
\end{aligned}
$$

which is (ii).

Lemma 2.7. [21] If $f$ and $g$ share $(0,0),(1,0),(\infty, 0)$ and $H \equiv 0$, then $f$ and $g$ share $(0, \infty),(1, \infty),(\infty, \infty)$.

Lemma 2.8. [23] If $f$ and $g$ share $\left(0, k_{1}\right),\left(1, k_{2}\right)$ and $\left(\infty, k_{3}\right)$, where $k_{1}, k_{2}, k_{3}$ are three positive integers satisfying $k_{1} k_{2} k_{3}>k_{1}+k_{2}+k_{3}+2$ and $f \not \equiv g$ then $\bar{N}(r, a ; f \mid \geq$ $2)+\bar{N}(r, a ; g \mid \geq 2)=S(r)$ for $a \in\{0,1, \infty\}$. 
Lemma 2.9. Suppose that $f$ and $g$ are two distinct nonconstant meromorphic functions that share $0,1, \infty C M$. Then $N(r, 0 ; f \mid \geq 2)+N(r, 1 ; f \mid \geq 2)+N(r, \infty ; f \mid \geq$ $2)=S(r, f)$, which is Lemma 4 in [20].

Lemma 2.10. [23] If $f$ and $g$ share $\left(0, k_{1}\right),\left(1, k_{2}\right)$ and $\left(\infty, k_{3}\right)$ where $k_{1}, k_{2}, k_{3}$ are three positive integers satisfying $k_{1} k_{2} k_{3}>k_{1}+k_{2}+k_{3}+2$ and $f \not \equiv g$ then for any $a(\neq 0,1, \infty), \bar{N}(r, a ; f \mid \geq 3)+\bar{N}(r, a ; g \mid \geq 3)=S(r)$.

Lemma 2.11. [13] If $f$ and $g$ share $\left(0, k_{1}\right),\left(1, k_{2}\right)$ and $\left(\infty, k_{3}\right)$ where $k_{1}, k_{2}, k_{3}$ are three positive integers satisfying $k_{1} k_{2} k_{3}>k_{1}+k_{2}+k_{3}+2$ and $f \not \equiv g$. Let $\alpha=\frac{f-1}{g-1}$ and $h=\frac{g}{h}$, then $\bar{N}(r, 0 ; \alpha)+\bar{N}(r, \infty ; \alpha)+\bar{N}(r, 0 ; h)+\bar{N}(r, \infty ; h)=S(r)$.

Lemma 2.12. [12] Let $f$ and $g$ be two distinct nonconstant meromorphic functions sharing $(0,0),(1,0),(\infty, 0)$. If $f$ is a bilinear transformation of $g$ then any one of the following holds:

(i) $f g \equiv 1$;

(ii) $(f-1)(g-1) \equiv 1$;

(iii) $f+g \equiv 1$;

(iv) $f \equiv c g,(v) f-1 \equiv c(g-1)$;

(vi) $[(c-1) f+1][(c-1) g-c]+c \equiv 1$;

where $c(\neq 0,1, \infty)$ is a constant.

Lemma 2.13. [13] Let $f$ and $g$ be two nonconstant meromorphic functions sharing $\left(0, k_{1}\right),\left(1, k_{2}\right)$ and $\left(\infty, k_{3}\right)$ where $k_{1}, k_{2}, k_{3}$ are three positive integers satisfying $k_{1} k_{2} k_{3}>k_{1}+k_{2}+k_{3}+2$. If $\lim \sup _{r \rightarrow \infty} \frac{N_{0}(r)}{T(r, f)}>\frac{1}{2}$, then $f$ is a bilinear transformation of $g$, where $N_{0}(r)$ is the counting function of the zeros of $f-g$ not containing the zeros of $f, f-1$ and $\frac{1}{f}$.

Lemma 2.14. If $f$ and $g$ share $\left(0, k_{1}\right),\left(1, k_{2}\right)$ and $\left(\infty, k_{3}\right)$ where $k_{1}, k_{2}, k_{3}$ are three positive integers satisfying $k_{1} k_{2} k_{3}>k_{1}+k_{2}+k_{3}+2$ and $f$ is not a bilinear transformation of $g$, then for any $a(\neq 0,1, \infty)$ each of the following holds:

(i) $T(r, f)+T(r, g)=\sum_{i=0,1, \infty} N(r, i ; f \mid=1)+N_{0}^{1}(r)+S(r)$;

(ii) $N(r, a ; f \mid \geq 3)=S(r, f), N(r, a ; g \mid \geq 3)=S(r, g)$;

(iii) $T(r, f)=N(r, a ; f \mid \leq 2)+S(r, f), T(r, g)=N(r, a ; g \mid \leq 2)+S(r, g)$;

(iv) $N(r, 0 ; f-g \mid g=\infty)+N(r, 0 ; f-g \mid f=\infty)=S(r)$;

(v) $N(r, 0 ; f-g \mid \geq 2)=S(r)$;

where $N_{0}^{1}(r)$ denotes the counting function of those simple zeros of $f-g$ which are not the zeros of $f(f-1)$ and $\frac{1}{f}$ and $N(r, 0 ; f-g \mid g=\infty)$ denotes the counting function of those zeros of $f-g$ which are poles of $g$.

Proof. Using Lemmas 2.8 and 2.9 the above Lemma can be proved in the line of the proof of Lemma 2.5 [11]. 
Lemma 2.15. [16] Let $f$ be a nonconstant meromorphic function and $R(f)=\frac{\sum_{i=0}^{m} a_{i} f^{i}}{\sum_{j=0}^{n} b_{j} f^{j}}$ be a nonconstant irreducible rational in $f$ with constant coefficients $a_{i}$ and $b_{j}$ satisfying $a_{m} \neq 0$ and $b_{n} \neq 0$. Then $T(r, R(f))=\max \{m, n\} T(r, f)+O(1)$.

Lemma 2.16. [24] Let $f_{1}$ and $f_{2}$ be two nonconstant meromorphic functions satisfying $\bar{N}\left(r, f_{j}\right)+\bar{N}\left(r, \frac{1}{f_{j}}\right)=S(r)(j=1,2)$. Then either $\bar{N}_{0}\left(r, 1 ; f_{1}, f_{2}\right)=S(r)$ or there exist two integers $s, t(|s|+|t|>0)$ such that $f_{1}^{s} f_{2}^{t} \equiv 1$, where $\bar{N}_{0}\left(r, 1 ; f_{1}, f_{2}\right)$ denotes the reduced counting function related to 1-points of $f_{1}$ and $f_{2}$ and $T(r)=$ $T\left(r, f_{1}\right)+T\left(r, f_{2}\right), S(r)=o\{T(r)\}(r \rightarrow \infty, r \notin E)$ depending only on $f_{1}$ and $f_{2}$.

Lemma 2.17. [24] Let $s$ and $t$ be relatively prime integers with $s>0$. Then $x^{s}-1$ and $x^{t}-c$ have one and only one common factor, where $c$ is a constant satisfying $c^{s}=1$.

Lemma 2.18. [13] Let $f$ and $g$ be two distinct nonconstant meromorphic functions sharing $\left(0, k_{1}\right),\left(1, k_{2}\right)$ and $\left(\infty, k_{3}\right)$ where $k_{1}, k_{2}, k_{3}$ are three positive integers satisfying $k_{1} k_{2} k_{3}>k_{1}+k_{2}+k_{3}+2$ and $N_{0}(r) \neq S(r, f)$. If $f$ is a fractional linear transformation of $g$, then $N_{0}(r)=T(r, f)+S(r, f)$. If $f$ is not a fractional linear transformation of $g$, then $N_{0}(r) \leq \frac{1}{2} T(r, f)+S(r, f)$ and $f$ and $g$ assume one of the following three relations:

(i) $f \equiv \frac{e^{(k+1) \gamma}-1}{e^{(k+1-s) \gamma}-1}, g \equiv \frac{e^{-(k+1) \gamma}-1}{e^{-(k+1-s) \gamma}-1}$;

(ii) $f \equiv \frac{e^{s \gamma}-1}{e^{(k+1) \gamma}-1}, g \equiv \frac{e^{-s \gamma}-1}{e^{-(k+1) \gamma}-1}$;

(iii) $f \equiv \frac{e^{s \gamma}-1}{e^{-(k+1-s) \gamma}-1}, g \equiv \frac{e^{-s \gamma}-1}{e^{(k+1-s) \gamma}-1}$;

where $\gamma$ is a nonconstant entire function, $s$ and $k(\geq 2)$ are positive integers such that $s$ and $k+1$ are relatively prime and $1 \leq s \leq k$.

\section{Proofs of the theorems}

Proof of Theorem 1.14. We first suppose that $H \not \equiv 0$. Let $N_{*}\left(r, 0 ; f^{\prime}\right)$ denote the counting function of those zeros of $f$ which are not the zeros of $f(f-1) \prod_{i=1}^{n}\left(f-a_{i}\right)$ where $a_{i}(\neq 0,1, \infty), i=1,2,3, . . n$, are distinct complex numbers. Also we define $N_{0}\left(r, 0 ; g^{\prime}\right)$ as the counting function those zeros of $g^{\prime}$ which are not the zeros of $g(g-1)$. Then from the second fundamental theorem we obtain using Lemma 2.5 and Lemma 2.6 with $k=1$, for any $n$ distinct complex numbers $a_{1}, a_{2}, a_{3}, . ., a_{n}$, different from $0,1, \infty$,

$$
\begin{aligned}
& (n+1) T(r, f)+T(r, g) \leq \bar{N}(r, 0 ; f)+\bar{N}(r, 1 ; f)+\bar{N}(r, \infty ; f)+\bar{N}(r, 0 ; g)+\bar{N}(r, 1 ; g) \\
& \quad+\bar{N}(r, \infty ; g)+\sum_{i=1}^{n} \bar{N}\left(r, a_{i} ; f\right)-N_{*}\left(r, 0 ; f^{\prime}\right)-N_{0}\left(r, 0 ; g^{\prime}\right)+S(r, f)+S(r, g)
\end{aligned}
$$




$$
\begin{aligned}
\leq & 2 \bar{N}(r, 0 ; f)+2 \bar{N}(r, \infty ; f)+\bar{N}_{*}(r, 0 ; f, g)+\bar{N}_{*}(r, \infty ; f, g)+\bar{N}_{g>2}(r, 1 ; f) \\
& +\bar{N}_{f>2}(r, 1 ; g)+\sum_{i=1}^{n} \bar{N}\left(r, a_{i} ; f\right)-N_{*}\left(r, 0 ; f^{\prime}\right)-N_{0}\left(r, 0 ; g^{\prime}\right)+T(r, g)-m(r, 1 ; g) \\
& +\bar{N}_{0}\left(r, 0 ; f^{\prime}\right)+\bar{N}_{0}\left(r, 0 ; g^{\prime}\right)+S(r, f)+S(r, g) \\
\leq & 2 \bar{N}(r, 0 ; f)+2 \bar{N}(r, \infty ; f)+\bar{N}(r, 0 ; f \mid \geq 2)+\bar{N}(r, \infty ; f \mid \geq 2)+\sum_{i=1}^{n} \bar{N}\left(r, a_{i} ; f\right) \\
& +\sum_{i=1}^{n} \bar{N}\left(r, a_{i} ; f \mid \geq 2\right)+\bar{N}_{*}(r, 1 ; f, g)+T(r, g)-m(r, 1 ; g)+S(r, f)+S(r, g) \\
\leq & 2 \bar{N}(r, 0 ; f)+2 \bar{N}(r, \infty ; f)+2 \bar{N}(r, 0 ; f \mid \geq 2)+2 \bar{N}(r, \infty ; f \mid \geq 2)+\sum_{i=1}^{n} N_{2}\left(r, a_{i} ; f\right) \\
& +T(r, g)-m(r, 1 ; g)+S(r, f)+S(r, g) .
\end{aligned}
$$

Thus since by Lemma 2.1, $S(r, f)=S(r, g)=S(r)$, we obtain from above

$$
(n+1) T(r, f) \leq 2 N_{2}(r, 0 ; f)+2 N_{2}(r, \infty ; f)+\sum_{i=1}^{n} N_{2}\left(r, a_{i} ; f\right)-m(r, 1 ; g)+S(r),
$$

from which we see that for arbitrary $\epsilon>0$ it holds

$$
3 \geq 2 \delta_{2}(0 ; f)+2 \delta_{2}(\infty ; f)+\sum_{i=1}^{n} \delta_{2}\left(a_{i}, f\right)+\liminf _{r \rightarrow \infty} \frac{m(r, 1 ; g)}{T(r, f)}-\epsilon .
$$

This being true for any distinct complex numbers $a_{i}(\neq 0,1, \infty), i=1,2,3, \ldots n$, we have

$$
3 \geq 2 \delta_{2}(0 ; f)+2 \delta_{2}(\infty ; f)+\sum_{a \neq 0, \infty, 1} \delta_{2}(a, f)+\liminf _{r \rightarrow \infty} \frac{m(r, 1 ; g)}{T(r, f)}-\epsilon .
$$

Since $\epsilon>0$ is arbitrary above leads to a contradiction to (1.6). Hence $H \equiv 0$. Therefore by Lemma 2.7 we see that $f, g$ share $(0, \infty),(1, \infty),(\infty, \infty)$. Thus Lemma 2.8 and Lemma 2.9 reduce the inequality 1.6 in the following:

$$
2 \delta_{1)}(0 ; f)+2 \delta_{1)}(\infty ; f)+\sum_{a \neq 0,1, \infty} \delta_{2)}(a ; f)+\liminf _{r \rightarrow \infty} \frac{m(r, 1 ; g)}{T(r, f)}>3 .
$$

Now suppose that $f$ is not a bilinear transformation of $g$. Then since $f, g$ share $(0, \infty),(1, \infty)$ and $(\infty, \infty)$ we have from (iii) of Lemma $2.13, \delta(a ; f)=\delta_{2)}(a ; f)=0$ for all $a \neq 0,1, \infty$. Therefore, from (3.1) we have

$$
N(r, 0 ; f \mid=1)+N(r, \infty ; f \mid=1)<\left(\frac{1}{2}-\varepsilon_{0}\right) T(r, f)+\frac{1}{2} m(r, 1 ; g) .
$$

Now using (i) of Lemma 2.13, from (3.2) we get

$$
T(r, f)+T(r, g)<\left(\frac{1}{2}-\varepsilon_{0}\right) T(r, f)+\frac{1}{2} m(r, 1 ; g)+N(r, 1 ; g \mid=1)+N_{0}(r) \text {. }
$$


That is,

$$
\begin{aligned}
T(r, f)+N(r, 1 ; g)+m(r, 1 ; g)< & \left(\frac{1}{2}-\varepsilon_{0}\right) T(r, f)+\frac{1}{2} m(r, 1 ; g)+N(r, 1 ; g \mid=1) \\
& +N_{0}(r),
\end{aligned}
$$

where $\varepsilon_{0}$ is sufficiently small positive number and hence $\lim \sup _{r \rightarrow \infty} \frac{N_{0}(r)}{T(r, f)}>\frac{1}{2}$. This, by Lemma 2.12 leads our assumption that $f$ is not a bilinear transformation of $g$ to a contradiction. Therefore $f$ must be a bilinear transformation of $g$ and one of the relations of Lemma 2.11 must be satisfied by $f$ and $g$. Suppose $f \not \equiv g$. We show that $f g \equiv 1$.

If $(f-1)(g-1) \equiv 1$ then $1, \infty$ are the evPs (exceptional values of Picard) of $f$. Therefore $1=\delta_{1)}(\infty, f)=\delta(1, f)$ and hence $0=\delta_{1)}(0, f)=\delta_{2)}(a, f)$ for $a \neq 0,1, \infty$. Since 1 is an evP of $f, N(r, 1 ; g)=S(r)$ and $f$ being a bilinear transformation of $g$, $T(r, f) \sim T(r, g)=m(r, 1 ; g)+S(r)$. Thus $2 \delta_{1)}(0 ; f)+2 \delta_{1)}(\infty ; f)+\sum_{a \neq 0,1, \infty} \delta_{2)}(a ; f)+$ $\liminf _{r \rightarrow \infty} \frac{m(r, 1 ; g)}{T(r, f)}=3$, contradicting (3.1). If $f+g \equiv 1$ then 0,1 are the evPs of $f$ giving $\delta_{1)}(0, f)=\delta_{1)}(1, f)=1$. Therefore, $2 \delta_{1)}(0 ; f)+2 \delta_{1)}(\infty ; f)+\sum_{a \neq 0,1, \infty} \delta_{2)}(a ; f)+$ $\liminf _{r \rightarrow \infty} \frac{m(r, 1 ; g)}{T(r, f)}=3$, as before contradicting (3.1). If $f \equiv c g$ then 1 and $c$ are evPs and $2 \delta_{1)}(0 ; f)+2 \delta_{1)}(\infty ; f)+\sum_{a \neq 0,1, \infty} \delta_{2)}(a ; f)+\liminf _{r \rightarrow \infty} \frac{m(r, 1 ; g)}{T(r, f)}=2$ contradicting (3.1). If $f-1 \equiv c(g-1)$, then 0 and $1-c$ are the evPs of $f$ which give $2 \delta_{1)}(0 ; f)+2 \delta_{1)}(\infty ; f)+\sum_{a \neq 0,1, \infty} \delta_{2)}(a ; f)+\liminf _{r \rightarrow \infty} \frac{m(r, 1 ; g)}{T(r, f)}=3$ contradicting (3.1). If $[(c-1) f+1][(c-1) g-c]+c \equiv 0$, then $\infty, \frac{1}{1-c}$ are evPs of $f$. Thus as before $2 \delta_{1)}(0 ; f)+2 \delta_{1)}(\infty ; f)+\sum_{a \neq 0,1, \infty} \delta_{2)}(a ; f)+\liminf _{r \rightarrow \infty} \frac{m(r, 1 ; g)}{T(r, f)}=3$ contradicting (3.1). However, if $f g \equiv 1,0$ and $\infty$ are the evPs of $f$ and we obtain $2 \delta_{1)}(0 ; f)+2 \delta_{1)}(\infty ; f)+\sum_{a \neq 0,1, \infty} \delta_{2)}(a ; f)+\liminf _{r \rightarrow \infty} \frac{m(r, 1 ; g)}{T(r, f)}=4$ which supports (3.1) and therefore $f g \equiv 1$. This completes the proof.

Proof of Theorem 1.15. Let $f$ be not a bilinear transformation of $g$. Therefore by (iii) of Lemma 2.13 we have $\delta_{2}(a, f)=\delta_{2)}(a, g)=0$ for $a \neq 0,1, \infty$. Thus (1.7) reduces to

$$
2 \delta_{1)}(0 ; f)+2 \delta_{1)}(\infty ; f)+\max \left\{\delta_{1)}(1 ; f), \delta_{1)}(1 ; g)\right\}>3 .
$$

Let $N_{0}^{*}(r)$ denote the counting function of those zeros of $f-g$ which are not the zeros of $g(g-1)$. Then $N_{0}^{*}(r)-N_{0}(r)\left(N_{0}(r)\right.$ is defined in Lemma 2.12 ) is the counting function of those zeros of $f-g$ which are the poles of $f$. So by (v) of Lemma 2.13 we obtain

$$
N_{0}^{*}(r)-N_{0}(r)=S(r)
$$

We now prove that $\bar{N}_{0}(r, 1 ; \alpha, h)=N_{0}(r)+S(r)$. To do this we consider the following cases.

Case 1. Let $z_{0}$ be a common simple zero of $f$ and $g$ such that $\alpha\left(z_{0}\right)=h\left(z_{0}\right)=1$. Since $h-1=\frac{g-f}{f}$, it follows that $z_{0}$ is a multiple zero of $f-g$. Hence by Lemma 2.9 
and (v) of Lemma 2.13, we see that the reduced counting function of the zeros of $f$ and $g$ for which $\alpha(z)=h(z)=1$ is $S(r)$.

Case 2. Let $z_{1}$ be a common simple 1-point of $f$ and $g$ such that $\alpha\left(z_{1}\right)=h\left(z_{1}\right)=1$. Since $\alpha-1=\frac{f-g}{g-1}$, it follows that $z_{1}$ is a multiple zero of $f-g$. Hence by Lemma 2.9 and (v) of Lemma 2.13 we see that the reduced counting function of those 1-points of $f$ and $g$ for which $\alpha(z)=h(z)=1$ is $S(r)$.

Case 3. Let $z_{2}$ be a common simple pole of $f$ and $g$ such that $\alpha\left(z_{2}\right)=h\left(z_{2}\right)=1$. Since $\alpha-1=\frac{f-g}{g-1}$ and $h-1=\frac{g-f}{f}$, it follows that $z_{2}$ is not a pole of $f-g$. Hence $z_{2}$ is a zero of $\alpha+h-2=\frac{(f-g)(f-g+1)}{f(g-1)}$ with multiplicity $\geq 2$ and so $z_{2}$ is a zero of $\alpha^{\prime}+h^{\prime}$. Also $\frac{\alpha^{\prime}}{\alpha}+\frac{h^{\prime}}{h}=\left(\alpha^{\prime}+h^{\prime}\right) \frac{\alpha+h-1}{\alpha h}-\frac{(\alpha-1) \alpha^{\prime}+(h-1) h^{\prime}}{\alpha h}$. Since $f$ is not a bilinear transformation of $g, \frac{\alpha^{\prime}}{\alpha}+\frac{h^{\prime}}{h} \not \equiv 0$. From the preceding identity, we see that $z_{2}$ is a zero of $\frac{\alpha^{\prime}}{\alpha}+\frac{h^{\prime}}{h}$. Now by Lemma 2.10, we get

$$
\begin{aligned}
N\left(r, 0 ; \frac{\alpha^{\prime}}{\alpha}+\frac{h^{\prime}}{h}\right) & \leq T\left(r, \frac{\alpha^{\prime}}{\alpha}+\frac{h^{\prime}}{h}\right) \\
& =N\left(r, \frac{\alpha^{\prime}}{\alpha}\right)+N\left(r, \frac{h^{\prime}}{h}\right)+S(r) \\
& =\bar{N}(r, 0 ; \alpha)+\bar{N}(r, \infty ; \alpha)+\bar{N}(r, 0 ; h)+\bar{N}(r, \infty ; h)+S(r) \\
& =S(r) .
\end{aligned}
$$

Therefore by Lemma 2.8 we see that the reduced counting function of the poles of $f$ and $g$ for which $\alpha(z)=h(z)=1$ is $S(r)$. Also by (v) of Lemma 2.13 we obtain $N_{0}^{*}(r)=\bar{N}_{0}^{*}(r)+S(r)$ and $N_{0}(r)=\bar{N}_{0}(r)+S(r)$.

Hence from above, by (3.3), we get

$$
\bar{N}_{0}(r, 1 ; \alpha, h)=N_{0}^{*}(r)+S(r)=N_{0}(r)+S(r) .
$$

Suppose that $N_{0}(r) \neq S(r)$. Then, $\bar{N}_{0}(r, 1 ; \alpha, h) \neq S(r)$. Then there exist integers $s, t$ such that $|s|+|t|>0$ and $\alpha^{s} h^{t} \equiv 1$ from which we obtain $(f-1)^{s} g^{t} \equiv$ $f^{t}(g-1)^{s}$. Since $f$ is not a bilinear transformation of $g, s \neq 0$ and $t \neq 0$ and $|s| \neq|t|$ and so $f, g$ share $0,1, \infty$ CM. Then $f$ and $g$ assumes one of the relations (i), (ii), (iii) of Lemma 2.17. If (i) holds then since $s, k+1$ are relatively prime $k+1, k+1-s$ are also relatively prime. Thus by Lemmas 2.14 and 2.16 we have $T(r, f) \sim \max \{k+1-1, k+1-s-1\} T\left(r, e^{\gamma}\right)=k T\left(r, e^{\gamma}\right)$. Also in this case $g=\frac{1-e^{(k+1) \gamma}}{\left\{1-e^{(k+1-s) \gamma}\right\} e^{s \gamma}}$ and hence by Lemmas 2.14 and 2.16 we get $T(r, g) \sim \max \{k+$ $1-1, k+1-1-s+s\} T\left(r, e^{\gamma}\right)=k T\left(r, e^{\gamma}\right)$. Arguing in same manner we can show that $T(r, f) \sim k T\left(r, e^{\gamma}\right)$ and $T(r, g) \sim k T\left(r, e^{\gamma}\right)$ if either (ii) or (iii) holds. Thus $T(r, f)=T(r, g)+O(1)$ and this gives $\delta_{1)}(1, f)=\delta_{1)}(1, g)$. Hence from (1.2) we obtain $\left(2-\varepsilon_{0}\right) T(r, f)>2 N(r, 0 ; f \mid=1)+2 N(r, \infty ; f \mid=1)+N(r, 1 ; f \mid=1)$, where $\varepsilon_{0}$ is a sufficiently small positive number. Combining above with (i) of Lemma 2.13 we have $N(r, 0 ; f \mid=1)+N(r, \infty ; f \mid=1)+N(r, 1 ; f \mid=1)+N_{0}(r)-\varepsilon_{0} T(r, f)>$ 
$2 N(r, 0 ; f \mid=1)+2 N(r, \infty ; f \mid=1)+N(r, 1 ; f \mid=1)$, and therefore

$$
N_{0}(r)>N(r, 0 ; f \mid=1)+N(r, \infty ; f \mid=1)+\varepsilon_{0} T(r, f) .
$$

Hence again from (i) of Lemma 2.13 and (3.5) we have $2 T(r, f)<2 N_{0}(r)+N(r, 1 ; f \mid=$ 1) $+\varepsilon_{0} T(r, f)$, that is $\left(1-\varepsilon_{0}\right) T(r, f)<2 N_{0}(r)$ which implies by Lemma 2.12 that $f$ is a bilinear transformation of $g$. This contradicts our assumption. Next suppose that $N_{0}(r)=S(r)$. Then (i) of Lemma 2.13 gives

$$
T(r, f)+T(r, g)=N(r, 0 ; f \mid=1)+N(r, \infty ; f \mid=1)+N(r, 1 ; f \mid=1)+S(r)
$$

Combining (3.5) and (3.6) we get

$$
\begin{aligned}
T(r, f)+T(r, g)< & {\left[\frac{1}{2}+\frac{1}{2} \max \left\{\delta_{1)}(1 ; f), \delta_{1)}(1 ; g)\right\}\right] T(r, f) } \\
& +N(r, 1 ; f \mid=1)+S(r)
\end{aligned}
$$

which implies $T(r, g)<N(r, 1 ; f \mid=1)+S(r)$ and therefore $T(r, g)<N(r, 1 ; g \mid=$ $1)+S(r)$ which is a contradiction. Therefore $f$ must be bilinear transformation of $g$. For the rest of the part of the proof we proceed exactly as Theorem 1.14.

Proof of Theorem 1.16. Since $f, g$ share $(\infty, 0)$ and $(0,1)$ we have $\bar{N}_{*}(r, \infty ; f, g)=$ $\bar{N}(r, \infty ; f)$ and $\bar{N}_{*}(r, 0 ; f, g)=\bar{N}(r, 0 ; f \mid \geq 2)$. We first suppose that $H \not \equiv 0$. Proceeding exactly as Theorem 1.14 we obtain using the second fundamental theorem and Lemma 2.5 and (ii) of Lemma 2.6

$$
\begin{aligned}
(n & +1) T(r, f)+T(r, g) \leq \bar{N}(r, 0 ; f)+\bar{N}(r, 1 ; f)+\bar{N}(r, \infty ; f)+\bar{N}(r, 0 ; g)+\bar{N}(r, 1 ; g) \\
& +\bar{N}(r, \infty ; g)+\sum_{i=1}^{n} \bar{N}\left(r, a_{i} ; f\right)-N_{*}\left(r, 0 ; f^{\prime}\right)-N_{0}\left(r, 0 ; g^{\prime}\right)+S(r) \\
\leq & 2 \bar{N}(r, 0 ; f)+2 \bar{N}(r, \infty ; f)+\sum_{i=1}^{n} \bar{N}\left(r, a_{i} ; f\right)+\sum_{i=1}^{n} \bar{N}\left(r, a_{i} ; f \mid \geq 2\right)+\bar{N}_{*}(r, 0 ; f, g) \\
& +\bar{N}_{*}(r, \infty ; f, g)+T(r, g)-m(r, 1 ; g)-\bar{N}_{L}(r, 1 ; g)-\bar{N}_{E}^{(3}(r, 1 ; f)+\bar{N}_{g>2}(r, 1 ; f) \\
& +\bar{N}_{f>2}(r, 1 ; g)+S(r) \\
\leq & 2 \bar{N}(r, 0 ; f)+3 \bar{N}(r, \infty ; f)+\bar{N}(r, 0 ; f \mid \geq 2)+\sum_{i=1}^{n} N_{2}\left(r, a_{i} ; f\right)+T(r, g)-m(r, 1 ; g) \\
& +\bar{N} g>2(r, 1 ; f)+\bar{N} f>2(r, 1 ; g)+S(r) \\
\leq & 2 \bar{N}(r, 0 ; f)+3 \bar{N}(r, \infty ; f)+\bar{N}(r, 0 ; f \mid \geq 2)+\sum_{i=1}^{n} N_{2}\left(r, a_{i} ; f\right)+T(r, g)-m(r, 1 ; g) \\
& +\bar{N}(r, 0 ; f \mid \geq 2)+\bar{N}(r, \infty ; f)+S(r),
\end{aligned}
$$


from which we obtain,

$$
(n+1) T(r, f) \leq 2 N_{2}(r, 0 ; f)+4 \bar{N}(r, \infty ; f)+\sum_{i=1}^{n} N_{2}\left(r, a_{i} ; f\right)-m(r, 1 ; g)+S(r) .
$$

Therefore, arguing as in the proof of Theorem 1.14, for arbitrary $\epsilon>0$ we obtain $5 \geq 2 \delta_{2}(0 ; f)+4 \Theta(\infty ; f)+\sum_{a \neq 0,1, \infty} \delta_{2}(a ; f)+\liminf _{r \rightarrow \infty} \frac{m(r, 1 ; g)}{T(r, f)}-\epsilon$, which contradicts (1.8). Thus $H \equiv 0$. The rest of the part of our proof can be carried out in the line of proof of the Theorem 1.14.

Proof of Theorem 1.17. First suppose that $f$ is not a bilinear transformation of $g$. Then by (iii) of Lemma 2.13, (1.9) and (1.10) reduce to

$$
2 \delta_{1)}(0 ; f)+2 \delta_{1)}(\infty ; f)+\delta_{1)}(1, f)+\delta_{1)}(1, g)>3
$$

and

$$
2 \delta_{1)}(0 ; g)+2 \delta_{1)}(\infty ; g)+\delta_{1)}(1, f)+\delta_{1)}(1, g)>3 \text {. }
$$

Then proceeding exactly as in the proof of Theorem 1.15 we obtain $\bar{N}_{0}(r, 1 ; \alpha, h)=$ $N_{0}^{*}(r)+S(r)=N_{0}(r)+S(r)\{$ see $(3.4)\}$ and then for $N_{0}(r) \neq S(r)$ we obtain as before $T(r, f) \sim T(r, g)$ and $\delta_{1)}(1, f)=\delta_{1)}(1, g)$. Thus from (3.7) and (3.8) we have $\delta_{1)}(0 ; f)+\delta_{1)}(\infty ; f)+\delta_{1)}(1, f)>\frac{3}{2}$, from which we obtain for sufficiently small $\varepsilon_{0}>0$,

$$
\bar{N}(r, 0 ; f \mid=1)+\bar{N}(r, \infty ; f \mid=1)+\bar{N}(r, 1 ; f \mid=1)<\left(\frac{3}{2}-\varepsilon_{0}\right) T(r, f) .
$$

Thus from (3.9) and (i) of Lemma 2.13 we see that $\left(\frac{1}{2}+\varepsilon_{0}\right) T(r, f)<N_{0}(r)$ which by Lemma 2.12 implies that $f$ is a bilinear transformation of $g$, contradicting our assumption. Next we suppose that $N_{0}(r)=S(r)$. Thus From (i) of Lemma 2.13 we obtain

$$
\begin{aligned}
T(r, f)+T(r, g) & =\bar{N}(r, 0 ; f \mid=1)+\bar{N}(r, \infty ; f \mid=1)+\bar{N}(r, 1 ; f \mid=1)+S(r) \\
& =\bar{N}(r, 0 ; g \mid=1)+\bar{N}(r, \infty ; g \mid=1)+\bar{N}(r, 1 ; g \mid=1)+S(r) \\
& \leq 3 T(r, g)+S(r),
\end{aligned}
$$

and hence $T(r, f) \leq 2 T(r, g)+S(r)$. Thus again from (i) of Lemma 2.13 we obtain $\frac{3}{2} T(r, f) \leq \bar{N}(r, 0 ; f \mid=1)+\bar{N}(r, \infty ; f \mid=1)+\bar{N}(r, 1 ; f \mid=1)+S(r)$, from which we obtain,

$$
3 \geq 2 \delta_{1)}(0 ; f)+2 \delta_{1)}(\infty ; f)+2 \delta_{1)}(1, f)
$$

Similarly we get,

$$
3 \geq 2 \delta_{1)}(0 ; g)+2 \delta_{1)}(\infty ; g)+2 \delta_{1)}(1, g) \text {. }
$$

Then the sum of (3.10) and (3.11) contradicts the sum of (3.7) and (3.8).

Hence $f$ must be a bilinear transformation of $g$. Then $f, g$ satisfy any one of the relations (i)-(vi) of Lemma 2.11. If $f, g$ satisfy any one of the conditions (i)-(iii), 
then any two of the values $0,1, \infty$ will be two exceptional values of Picard(evP) of $f$ and $g$ and hence $0=\sum_{a \neq 0,1, \infty} \delta_{2)}(a ; f)=\sum_{a \neq 0,1, \infty} \delta_{2)}(a ; g)$ and

$$
\begin{aligned}
& 2 \delta_{1)}(0 ; f)+2 \delta_{1)}(\infty ; f)+\delta_{1)}(1, f)+\delta_{1)}(1, g)>3, \\
& 2 \delta_{1)}(0 ; g)+2 \delta_{1)}(\infty ; g)+\delta_{1)}(1, f)+\delta_{1)}(1, g)>3,
\end{aligned}
$$

which imply (1.9) and (1.10). Similarly if $f, g$ satisfy any one of (iv)-(vi), then $1, c ; 1, \frac{1}{c}$ or, $0,1-c ; 0,1-\frac{1}{c}$ or $\infty, \frac{1}{1-c} ; \infty, \frac{c}{c-1}$ are the respective evPs of $f$ and $g$ from which we obtain (1.9) and (1.10) and therefore the conclusion of our Theorem is true.

Acknowledgment: Authors are thankful to the referee for his/her valuable comments and suggestions. Authors would also like to thank Abhijit Banerjee, Associate Professor of Mathematics, University of Kalyani for his help to prepare the manuscript.

\section{REFERENCES}

[1] A. Banerjee, Meromorphic functions that share three values with finite weight, Ann. Polon. Math. 91(1) (2007), 5-69.

[2] W. K. Hayman, Meromorphic Functions, Clarendon Press, Oxford (1964).

[3] G. G. Gundersen, Meromorphic functions that share three or four values, J. London Math. $\operatorname{Soc}(2), 20$ (1979), 457-466.

[4] I. Lahiri, Weighted value sharing and uniqueness of meromorphic functions, Complex Var. Vol.' 46 (2001), 241-253.

[5] I. Lahiri, Weighted sharing and uniqueness of meromorphic functions, Nagoya Math. J. 161 (2001), 193-206.

[6] I. Lahiri, Weighted sharing of three values and uniqueness of meromorphic functions, Kodai Math. J. 24 (2001), 421-435

[7] I. Lahiri, Weighted sharing and a result of Ozawa, Hokkaido Math. J. 24 (2001), 421-435.

[8] I. Lahiri, On a result of Ozawa concerning uniqueness of meromorphic functions, J. Math. Anal. Appl. 271 (2002), 206-216.

[9] I. Lahiri, On a result of Ozawa concerning uniqueness of meromorphic functions II, J. Math. Anal. Appl. 283 (2003), 66-76.

[10] I. Lahiri, Meromorphic functions sharing three values, Southeast Asian Bull. Math. 26 (2003), 961-966

[11] I. Lahiri, Characteristic functions of meromorphic functions sharing three values with finite weights, Complex Var. Theory Appl. 50(1) (2005), 69-78.

[12] I. Lahiri and A. Sarkar, On a uniqueness theorem of Tohge, Arch. Math. (Basel) 84(5) (2005), 461-469.

[13] I. Lahiri and P. Sahoo, Uniqueness of meromorphic function sharing three weighted values, Bull. Malayasian Math. Soc. (2)31(1) (2008), 67-75.

[14] X. M. Li and H. X. Yi, On uniqueness theorem of meromorphic function concerning weighted sharing of three values, Bull. Malayasian Math. Soc. (2)33(1) (2010), 1-67.

[15] C. Meng, On a result of Ozawa and uniqueness of meromorphic function, Bull. Malayasian Math. Soc. (2)31(1) (2008), 4-56.

[16] A. Z. Mohonko, On the Nevanlinna characteristic of some meromorphic functions, Funct. Anal. Appl. 14 (1971), 83-87.

[17] M. Ozawa, Unicity theorems for entire functions, J. Anal. Math. 30 (1976), 411-420. 
[18] H. Ueda, Unicity theorems for meromorphic or entire functionsII, Kodai Math. J. 6 (1983), 26-36.

[19] H. X. Yi, Meromorphic functions that share two or three values, Kodai Math. J. 13 (1990), $363-372$.

[20] H. X. Yi, Unicity theorems for meromorphic functions that share three values, Kodai Math. J. 18 (1995), 300-314.

[21] H. X. Yi, Meromorphic functions that share three values, Bull. Hong Kong Math. Soc. 2 (1998), 679-688.

[22] H. X. Yi and Thamir C Alzahari, Weighted value sharing and a question of I. Lahiri, Complex Var Theory Appl. 49(15) (2004), 1063-1078.

[23] H. X. Yi, Meromorphic function with weighted sharing of three values, Complex Var. Theory Appl. 50(12) (2005), 923-934.

[24] Q. C. Zhang, Meromorphic functions sharing three values, Indian J. Pure and Appl. Math. 30(7) (1999), 667-682.

[25] Q. C. Zhang, On the results of Lahiri concerning uniqueness of meromorphic functions, J. Math. Anal. Appl. 318 (2006), 707-725.

${ }^{1}$ Department of Mathematics,Kandi Raj College,

KANDI, MURSHIDABAD,

West BengAL, INDIA-742137.

E-mail address: arindam_ku@rediffmail.com

${ }^{2}$ Department of Mathematics,

ACADEmy of TeChNology,

West BengaL, InDIA-712121.

E-mail address: paulomi.chattopadhyay@rediffmail.com 